\title{
A new method for MR grayscale inhomogeneity correction
}

\begin{abstract}
Intensity inhomogeneity is a smooth intensity change inside originally homogeneous regions. Filter-based inhomogeneity correction methods have been commonly used in literatures. However, there are few literatures which compare effectiveness of these methods for inhomogeneity correction. In this paper, a new filter-based inhomogeneity correction method is proposed and the effectiveness of the proposed method and other filter-based inhomogeneity correction methods are compared. The methods with different kernel sizes are applied on MRI brain images and the quality of inhomogeneity correction of different methods are compared quantitatively. Experimental results show the proposed method in a kernel size of $20 * 20$ performs almost better than or equal the performance of other methods in all kernel sizes.
\end{abstract}

Keyword: Inhomogeneity correction; Max filter; Brain MRI 